\title{
Pointing with Fingers, Hands and Arms for Wearable Computing
}

\author{
Ian Oakley \\ LabUSE \\ University of Madeira \\ Funchal \\ 9000-390, Portugal \\ ian@uma.pt
}

\section{John Sunwoo}

Electronics and Telecommunications

Research Institute

1 Gajeong-dong, Yuseong-gu

Daejeon

Korea

bistdude@etri.re.kr

\section{Il-Yeon Cho}

Electronics and Telecommunications

Research Institute

1 Gajeong-dong, Yuseong-gu

Daejeon

Korea

iycho@etri.re.kr

Copyright is held by the author/owner(s).

CHI 2008, April 5 - April 10, 2008, Florence, Italy

ACM 978-1-60558-012-8/08/04.

\begin{abstract}
Pointing is a fundamental enabling operation for

human-computer interaction across a broad spectrum

of scenarios. The paper presents a study exploring how

to develop a pointing system for truly wearable, rather

than hand-held, computing. It describes a Fitts' law

study of pointing based on motions in free-space

captured using an inertial sensor pack. It compares

performance when the pack is held in the hand,

mounted on the back of the hand and finally on the

wrist. The results show a significant, but numerically small, advantage in using the hands over using the upper arm only. This suggests that for wearable tasks where pointing is relatively infrequent a wrist based sensor pack may well be sufficient to enable effective and usable interaction.
\end{abstract}

\section{Keywords}

Fitts' Law, wearable devices, motion input

\section{ACM Classification Keywords}

H.5.2 [Information Interfaces and Presentation]: User

Interfaces-Input devices and strategies.

\section{Introduction}

Pointing is the dominant interaction technique employed by everyday users in order to issue 
commands to computers; it fundamentally underlies graphical user interfaces. Attached to almost every computer, pointing devices are truly ubiquitous and their flexibility, adaptability, expressiveness, power and ease of use remains currently unmatched. Reflecting their importance, pointing tasks have received an extremely large amount of attention by the research community [e.g. 3, 9].

Largely through the enabling technology of touch screens, the pointing metaphor has successfully transitioned to mobile device form factors and can now be found on high end mobile phones. In many ways, it is also a good candidate for wearable computing applications. Many such systems and motivating scenarios involve either an external graphical user interface (such as a remote screen [e.g. 7]), or one worn on the body (such as a head-mounted display [e.g. 8]) and are therefore able to present the relatively rich $2 \mathrm{D}$ graphical feedback pointing interfaces require. However, one fundamental issue with pointing for wearable computing is that the conventional devices - the mouse and trackpad/touchscreen - are not well matched to the wearable domain. Both these conventional technologies rely on the presence of a surface to point against, something likely to be absent in a wearable scenario.

Diverse designs have been constructed as solutions to this problem. They can be broadly split into attempts to monitor the position of a device held in the hand (a gyro mouse [7], or laser pointer are good examples of such approaches) or attempts to support stylus or finger gestures against a sensor surface mounted on the body [10]. While this approach can yield good performance, the input techniques it supports are hand-held rather than truly wearable. This difference is more than simply conceptual as many wearable computing domains, such as systems to support medical personal (whose hands must remain sterile [13]) or skilled maintenance tasks [1] inherently rely on the hands being free. In many domains wearable technology is desirable solely because it offers the freedom for users' hands to be engaged in other tasks.

The obvious solution to this problem is to develop a pointing device which relies on movements of another part of the body. Although many such devices (e.g. involving the feet or eyes [2]), appear in the literature, they tend to assume a seated posture or require substantial external sensing equipment. This makes them unsuitable for wearable computing scenarios.

One approach that may be effective is to use arm motions (rather than hand) to control pointing. This has the advantage of being easily available in most wearable scenarios, but has received little attention in the literature, in part due to influential work by Card et al [3]. In this paper, the authors argued that the fingers play an important role in pointing tasks and that a device which better takes advantage of their dexterity could potentially outperform the mouse. No such device has appeared, but Zhai et al [12] gave credence to these claims with a well-grounded investigation of user performance in a 6 DOF input task. This work showed that a device manipulated by the fingers offered a clear advantage over one simply attached to the palm of the hand by a band.

Given the utility of pointing to wearable computing tasks, the goal of this paper is to revisit this issue but focus on simpler operations. It seeks to explore 


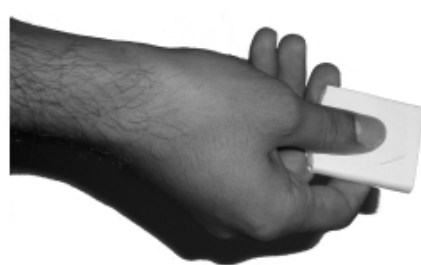

(a)

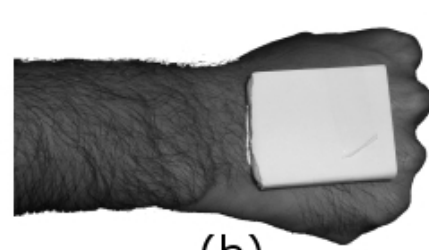

(b)

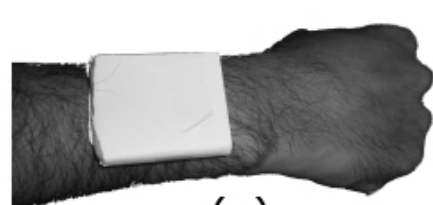

(c)

Figure 1. Three sensor positions used in study. White rectangle indicates Held (a), Hand-back (b) and Wrist (c). Sensor mounting materials are not shown. performance differences in elementary pointing tasks in a wearable context where the input device (an inertial sensor pack) is held in the hand, mounted on the hand or mounted on the wrist. It seeks a concrete answer to the question of whether the hands and fingers need to be involved in the kind of basic pointing tasks that compose GUI operation. If acceptable performance can be gained with an entirely worn sensor pack, this paper argues this is a much more appropriate solution for many wearable computing domains.

\section{Study}

The goal of this study was to investigate pointing

performance originating from movements in free space; an inertial sensor pack was used to control the pointing. A Fitts' law [5] model was adopted and three conditions were compared. In one the participants held the sensors in their hands. In the other two it was physically mounted on the back of their hand (using a tight glove) and on the dorsal surface of their wrist (using a sport strap designed to hold an MP3 player).

Subsequently these conditions are referred to as Held Hand-back and Wrist. They are illustrated in Figure 1.

\section{Participants}

12 participants took part in this study. Six were male, six female with an average age of 29 . They were sampled opportunistically via the snowball method and included research and administrative staff from our institute and their acquaintances. Only one was left handed and none reported any physical impairment other than (corrected) vision. The study took between 30 minutes and 1 hour and subjects were paid approximately 20 USD dollars. None had more than passing experience with motion input devices.
Fitts' Law Parameters

Fitts' law is a predictive mathematical model for describing the relationship between the size and distance of a target and the time and accuracy with which it can be reached. It is a part of ISO 9241-9 (which details procedures for evaluating pointing devices). See Soukoreff and Mackenzie [9] for an account of current best practices regarding its use; this paper does not attempt to fully describe Fitts' law procedures and interested parties are referred to this more comprehensive text for full information. It is sufficient to note that Fitts' law allows the calculation of throughput (in bits/second) for a given set of input device configurations, and therefore enables them to be easily compared. Furthermore Fitts' studies are based on the performance of rapid targeting tasks to targets with different sizes (or widths, W) and at different distances (D). These two parameters are combined to lead to an Index of Difficulty (ID) for each targeting operation.

The study reported here used three values of $W$ and $D$ (expressed in screen pixels). All targets were round and with a diameter $W$ of 20,40 or 80 . The values of $D$ were 80,160 and 320 . This is a common Fitts' law configuration leading to 9 different ID values between 1 and 4.08. An experimental block was defined as 25 targeting operations with a given ID value, arranged so that participants moved around the spokes of a virtual wheel. This design is part of the ISO standard and ensures that subjects engage in targeting motions in all directions. There were 9 possible experimental blocks.

\section{Experimental Design and Measures}

The study used a repeated measures design among its three main conditions of Held, Hand-back and Wrist; 
there were 6 order conditions each completed by 2 subjects. Each condition was composed of 3 repetitions of each of the 9 experimental blocks (delivered in a random order). The first of these repetitions was always treated as practice and the data was not included in the analysis. The experimental structure can be summarized as: 25 trials by 9 experimental blocks (ID values) by 3 repetitions (first repetition not analyzed) by 3 conditions by 12 subjects for a total of 24300 trials (of which 16200 were analyzed). The experimental measures were task completion time, error rate and final selection position (used to adjust the Fitts' law calculations to include error).

\section{Materials and Procedures}

The experimental software was implemented in $\mathrm{C}++$ on Microsoft Windows XP. Movements were captured from users with an X-Sens MTi sensor pack [11]. This provides tri-axial readings from gyroscopes (which measure rotational velocity) and accelerometers (which measure linear accelerations) at $100 \mathrm{~Hz}$. These two data were combined to control cursor position. The accelerometer readings were continually monitored to detect when the sensor pack was relatively stationary, and the direction of the constant $1 \mathrm{G}$ vector of gravity recorded at such moments. This vector was then used to offset the gyroscopic readings such that, irrespective of the orientation of the sensor pack, a movement by the user to the left, right, top or bottom resulted in an appropriate on-screen cursor movement. Thus users were able to alter their postures freely during the experiment and retain spatially coherent control of the device. This approach was adopted to support more natural motions. All participants held or wore the sensor pack on their dominant arms throughout the study.
The visual display was presented on a 24 inch flat panel, with the resolution set such that a one pixel size corresponded to approximately $0.5 \mathrm{~mm}$. Thus the visual size of the targets ranged between 1 and $4 \mathrm{~cm}$, while the distances between them were spread over 4,8 and $16 \mathrm{~cm}$. The display was approx one meter from the participants standing positions and set slightly beneath eye-level. In each trial the visual display consisted of an arrow-like cursor and a plain black circle indicating the target area in an otherwise blank screen. When intersected by the cursor, the target circle turned red.

Trials in the study were initiated by the press of a button and terminated by its release. A break, requiring an additional button press and release to bypass, was enforced between each trial. All button presses were issued with a dedicated hardware button held in the non-dominant (and otherwise unoccupied) hand, as this approach seemed to offer the least chance of

confounding data between the worn and held conditions. This discrete task was chosen as one weakness of freemotion cursor control is that, if a long series of movements is executed, the body can end up in an inconvenient posture. The continual breaks between the trials allow the users to complete a trial then return to a neutral position before starting the next one. This process is analogous to clutching, or lifting and replacing, the mouse on the table top in order to return to a more comfortable physical position without affecting the cursor.

Participants were left alone in a quiet office for the bulk of the experiment. During the first few practice trials an experimenter remained with them to clarify any issues with the procedures and returned to remount the sensor pack in the different body locations as the 


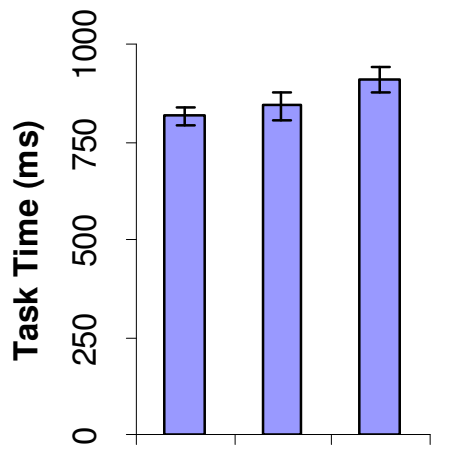

Held Hand- Wrist

Back

Condition

Figure 2. Task time (bars show standard error).

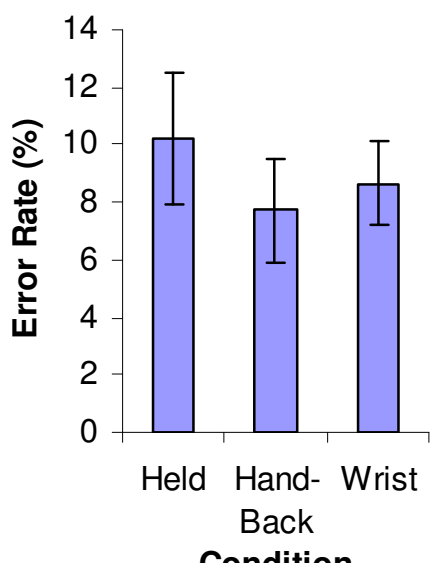

Figure 3. Error rate (bars show standard error). experiment demanded. Participants were told they could rest at any time when not directly performing a trial and explicitly encouraged to at the end of every set of 9 experimental blocks.

\section{Hypotheses}

The experimental hypotheses were that performance would degrade throughout the three conditions: best performance would appear in the Held condition and worst in the Wrist condition. The experiment was intended to explore the magnitude of these changes to better inform the design of wearable pointing systems.

Results

The data was initially subjected to standard procedures to remove outliers (defined as trials in which the task completion time was more than three standard deviations from the mean, or which was less than 50 $\mathrm{ms}$ in duration). A total of 384 (2.3\%) trials were discarded. The task completion time and error data from the remaining 15816 trials are presented in Figures 2 and 3. One-way repeated measures ANOVAs revealed significant differences in time $(F(2,11)=4.9$, $\mathrm{p}<0.05)$ but not errors $(F(2,11)=2.2, p=0.13)$. Posthoc t-tests including Bonferroni confidence interval adjustments showed the Held condition performed better than the Wrist condition $(p<0.05)$. The data were also subjected to a standard ISO 9241-9 Fitts' law analysis. This process involves adjusting the accuracy of the timing data (based on the scatter plot of the end-points) and producing a revised set of ID values. These are then used to calculate the throughput values (via a formulation known as the mean of means) which are shown in Figure 4. A one-way repeated measures ANOVA on these data showed a significant difference ( $F$ $(2,11)=6.891, p<0.005)$ which was born out by post- hoc t-tests showing that the Held condition offered advantages over the Wrist condition $(p<0.01)$.

\section{Discussion}

The results of the study show that there are gains to be made by involving the dexterity of the wrist and fingers in a pointing operation: respectively a 10 and 13 percent improvement in throughput was achieved over that recorded with from motions of a sensor pack mounted on the wrist. Examining the raw data, these differences can be attributed to changes in the task completion time (which shows a similar pattern) rather than the error rate.

The relatively small magnitude of these differences is encouraging. Although pointing performance (in a simple atomic task) is impaired with a wrist mounted sensor pack, it remains achievable both rapidly and accurately. Given the relatively novelty of purely wristbased input, it is also possible that participants may also improve with experience, eroding the observed differences. This has important implications for wearable computing system designers considering how best to integrate pointing operations. If a system

depends on complex, continuous pointing interactions, then clearly taking advantage of the dexterity of the hands is important. However, if a system is likely to feature only sporadic, infrequent or isolated pointing tasks then the convenience of completely freely the hands from pointing may well outweigh the relatively minor reductions in performance.

Conclusions and Future Work

This work is an initial attempt to explore how to best develop a truly wearable (rather than handheld) pointing system. It suggests that although there are 


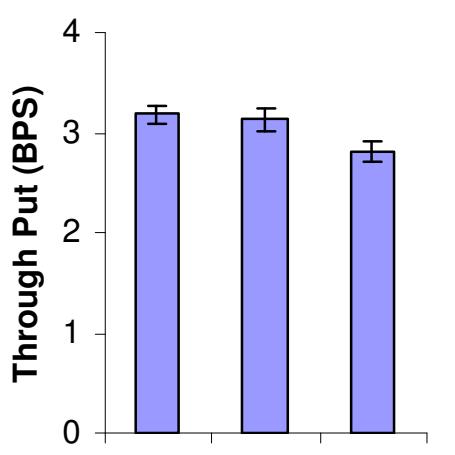

Hand Hand- Wrist

Back

Condition

Figure 4. Throughput (bars show standard error). advantages to using the hands, pointing performance using free-motions captured from the wrist remains

both rapid and accurate. However, many aspects

require further exploration. For example, the

movements in this study were delineated by a button

held in the user's non-dominant hand; additional effort

should be made to develop a hands-free solution to this problem. A further key issue is the reduction in the ease of performance of free-motion input during other tasks, such as walking [14]. It may also be interesting to explore the performance of gesture input (rather than pointing) between worn and held sensor packs [4]

In conclusion, pointing tasks will remain important as wearable computing applications develop. This work highlights an unexplored trade off between the expressivity of such movements and the intrusiveness of the equipment required to make them. For the development of truly wearable computing, this issue deserves further attention.

\section{Acknowledgements}

This work was supported by the IT R\&D program of the Korean MIC and IITA (2005-S-065-03, Development of Wearable Personal Station).

\section{References}

[1] Bo, G., Lorenzon, A., Chevassus, N. and Blondel V. Wearable Computing and Mobile Workers. 4th Int. Forum on Applied Wearable Computing (2006).

[2] Calhoun, G.L. and McMillan, G.R. Hands-free input devices for wearable computers. In 4th Symposium on Human Interaction with Complex Systems (1998).

[3] Card, S. K., Mackinlay, J. D., and Robertson, G. G. A morphological analysis of the design space of input devices. ACM Transactions on Information Systems 9, 2 (1991), 99-122.
[4] Cho, I , Sunwoo, J Son, $Y$, Oh, $M$, Lee, $C$ Development of a Single 3-axis Accelerometer Sensor Based Wearable Gesture Recognition Band. In Proceedings of Ubiquitous Intelligence and Computing, Hong Kong (2007)

[5] Fitts, P.M. The information capacity of the human motor system in controlling the amplitude of movement. Journal of Exp. Psychology 47, 6 (1954), 381-391.

[6] ISO, 2002. Reference Number: ISO 9241-

9:2000(E). Ergonomic requirements for office work with visual display terminals (VDTs)-Part 9-Requirements for non-keyboard input devices (ISO 9241-9) (2002).

[7] MacKenzie, I. S., \& Jusoh, S. (2001). An evaluation of two input devices for remote pointing. In Eighth IFIP International Conference on Engineering for HumanComputer Interaction (2001).

[8] Spitzer, M.B., Rensing, N.M., McClelland, R. and Aquilino, P. Eyeglass-Based Systems For Wearable Computing. Int. Symp. on Wearable Computing (1997)

[9] Soukoreff, R.W. and MacKenzie, S. Towards a standard for pointing device evaluation, perspectives on 27 years of Fitts' law research in HCI. Int. J. of Human Computer Studies 61 (2004), 751-789.

[10] Thomas, B.H., Grimmer, K., Zucco, J. and Milanese, S. Where does the mouse go? Personal and Ubiquitous Computing 6, 1 (2002), 97-112.

[11] XSens, http://www.xsens.com/

[12] Zhai, S., Milgram, P. and Buxton W. The Influence of Muscle Groups on Performance of Multiple Degree-ofFreedom Input. In $\mathrm{CHI}^{\prime} 96, \mathrm{ACM}$ Press, 308-315.

[13] Zinnen, A., Schiele, B. and Ziegert, T. Browsing patient records during ward rounds with a body worn gyroscope. In 11th IEEE Int. Symp. on Wearable Computers (2007).

[14] Zucco, J.E., Thomas, B.H. and Grimmer K. Evaluation of Four Wearable Computer Pointing Devices for Drag and Drop Tasks when Stationary and Walking. In 10th Int. Symp. on Wearable Computers (2006). 
